Published in final edited form as:

ACS Synth Biol. 2020 January 17; 9(1): 10-18. doi:10.1021/acssynbio.9b00347.

\title{
Point-of-Use Detection of Environmental Fluoride via a Cell-Free Riboswitch-Based Biosensor
}

\author{
Walter Thavarajah ${ }^{1,3,4}$, Adam D. Silverman ${ }^{1,3,4}$, Matthew S. Verosloff ${ }^{2,3,4}$, Nancy Kelley- \\ Loughnane $^{5}$, Michael C. Jewett ${ }^{1,3}$, Julius B. Lucks ${ }^{1,3,4,{ }^{*}}$ \\ 1 -Department of Chemical and Biological Engineering, Northwestern University, 2145 Sheridan \\ Rd, Evanston, IL, 60208, USA \\ 2 -Interdisciplinary Biological Sciences Graduate Program, Northwestern University, 2204 Tech \\ Drive, Evanston, IL, 60208, USA \\ 3 -Center for Synthetic Biology, Northwestern University, 2145 Sheridan Rd, Evanston, IL, 60208, \\ USA \\ 4 -Center for Water Research, Northwestern University, 2145 Sheridan Rd, Evanston, IL, 60208, \\ USA \\ 5 -Materials and Manufacturing Directorate, Air Force Research Laboratory, Wright-Patterson Air \\ Force Base, Ohio 45433, United States
}

\begin{abstract}
Advances in biosensor engineering have enabled the design of programmable molecular systems to detect a range of pathogens, nucleic acids, and chemicals. Here, we engineer and field-test a biosensor for fluoride, a major groundwater contaminant of global concern. The sensor consists of a cell-free system containing a DNA template that encodes a fluoride-responsive riboswitch regulating genes that produce a fluorescent or colorimetric output. Individual reactions can be lyophilized for long-term storage and detect fluoride at levels above 2 parts per million, the
\end{abstract}

\footnotetext{
* To whom correspondence should be addressed, jblucks@ @orthwestern.edu. Author Contributions

Conceptualization, W.T., A.D.S., M.C.J., \& J.B.L.; Data Curation, W.T., A.D.S., \& M.S.V.; Formal Analysis, W.T \& A.D.S.;

Investigation, W.T., A.D.S, \& M.S.V.; Methodology, W.T., A.D.S., M.S.V., \& J.B.L.; Project administration, W.T. \& J.B.L.; Validation, W.T., A.D.S., \& M.S.V.; Funding acquisition, N.K., M.C.J., \& J.B.L.; Writing - original draft, W.T., A.D.S., \& J.B.L.; Writing review \& editing, W.T., A.D.S., M.S.V., M.C.J., \& J.B.L.

Competing Interests Statement

The authors have submitted one provisional patent application (U.S. Patent Application Serial No. 62/813,368) for the technologically important developments included in this work. J.B.L is a cofounder of Stemloop, Inc. J.B.L.'s interests are reviewed and managed by Northwestern University in accordance with their conflict of interest policies.

Data Availability

All source data for main and SI figures was deposited open access in Northwestern's Arch database (https:// arch.library.northwestern.edu). Data can be accessed via https://doi.org/10.21985/N2RJ64.

Plasmid Availability

All plasmids are being deposited in Addgene with accession numbers 128809 - 128811.

Supporting Information

Supplemental figures S1-S8, supplemental tables S1 and S2, supplemental video, and supplemental experimental design spreadsheet.

Publisher's Disclaimer: This document is the Accepted Manuscript version of a Published Work that appeared in final form in $A C S$ Synthetic Biology copyright $\odot$ American Chemical Society after peer review and technical editing by the publisher. To access the final edited and published work see http://pubs.acs.org/articlesonrequest/AOR-wA85EJP2TRNWv9zeU5mN.
} 
Environmental Protection Agency's most stringent regulatory standard, in both laboratory and field conditions. Through onsite detection of fluoride in a real-world water source, this work provides a critical proof-of-principle for the future engineering of riboswitches and other biosensors to address challenges for global health and the environment.

\section{Keywords}

Riboswitches; biosensor; cell-free systems; diagnostics; water quality; field use

Safe drinking water availability is an important contributor to public welfare ${ }^{1}$. However, safe water sources are not available to a large portion of the globe, with an estimated 3 billion people using water from either an unsafe source or a source with significant sanitary risks ${ }^{2}$. One particularly dangerous contaminant is fluoride, which leaches into groundwater from natural sources. Long-term exposure to fluoride concentrations above 2 parts per million (ppm) can cause dental and skeletal fluorosis, heavily burdening communities in resourcelimited settings ${ }^{3}$. Though largescale remediation strategies are available, they are resourceintensive and difficult to deploy ${ }^{3,4}$. This problem is compounded by the reliance of goldstandard sensing methods on expensive analytical equipment, making detection difficult in areas with the greatest need ${ }^{4}$. While many emerging fluorescent and colorimetric chemical fluoride sensors exist, these either require supplementary imaging equipment or utilize toxic organic solvents, hampering their use in real-world conditions ${ }^{5}$. To facilitate targeted remediation and empower affected individuals, there is a pressing need for a more practical, rapid, and field-deployable solution to monitor the presence of fluoride in water.

Because they can be lyophilized and rehydrated on-demand, cell-free expression (CFE) systems have recently become a promising platform for field-deployable molecular diagnostics $^{6-9}$. These systems typically consist of cellular gene expression machinery along with the required buffers, energy sources and co-factors necessary to support gene expression from added DNA templates ${ }^{10}$. Unlike whole cells, cell-free platforms offer an open, easily tunable reaction environment, expediting the design process for genetically encoded programs ${ }^{10}$. Furthermore, they circumvent the analyte toxicity, host mutation, and biocontainment concerns limiting cellular sensors ${ }^{11}$.

We sought to leverage the advantages of cell-free biosensing platforms to create a new approach for monitoring for the presence of fluoride in water using a fluoride-responsive riboswitch that regulates the expression of the $\mathrm{CrcB}$ fluoride efflux pump in Bacillus cereus $^{12}$. By configuring the $B$. cereus $c r c B$ fluoride riboswitch to control the transcription of downstream reporter genes ${ }^{13}$, we show that a cell-free gene expression system can activate both protein and RNA reporter expression in the presence of fluoride. With an enzymatic colorimetric reporter, we demonstrate detection of fluoride concentrations at the Environmental Protection Agency (EPA) Secondary Maximum Contaminant Level of 2 $\mathrm{ppm}^{14}$. Notably, these cell-free biosensors showed more accurate sensing with a lower limit of detection than several tested commercially available consumer fluoride testing kits. We also demonstrate that our fluoride biosensor can be lyophilized for long-term storage and distribution, allowing us to detect fluoride in unprocessed groundwater obtained and tested 
onsite in Costa Rica. This work exemplifies the potential of riboswitches as practical biosensing tools and helps lay the foundation for utilizing cell-free biosensing systems in rapid and field-deployable water quality diagnostics to address pressing challenges in global health.

\section{Results}

\section{Fluoride riboswitch control of reporter expression in cell-free reactions}

Our point-of-use diagnostic consists of a cell-free system containing a fluoride biosensor DNA template that can be lyophilized and stored. Rehydration activates the biosensor, which encodes the fluoride riboswitch and a reporter gene that produces a detectable output if fluoride is present (Figure 1A, B). As a starting point, we sought to characterize the regulatory activity of the $B$. cereus $\operatorname{crc} B$ riboswitch in the cell-free reaction environment. Previous characterization of the riboswitch's cotranscriptional folding mechanism (Figure 1B) confirmed that it functions with $E$. coli RNA polymerase ${ }^{13}$, allowing us to use it in $E$. coli cell-free extract. We therefore constructed a reporter plasmid containing the riboswitch sequence followed by a strong ribosome binding site (RBS) and the coding sequence of the reporter protein superfolder green fluorescent protein (sfGFP), all placed downstream of a constitutive E. coli $\sigma^{70}$ promoter (all plasmid details in Supplemental Table 1).

After optimizing the level of $\mathrm{Mg}^{2+}$ within the reaction conditions for riboswitch performance (Supplemental Figure 1), we determined the fluoride sensor's dose-response to fluoride by titrating across a range of $\mathrm{NaF}$ concentrations. All tested conditions caused a measurable increase in expression over the OFF state, with activation seen at $\mathrm{NaF}$ concentrations as low as $0.1 \mathrm{mM}$ (Figure 1C, green line and inset). This threshold is important, since $0.1 \mathrm{mM} \mathrm{NaF}$ is equivalent to the EPA's 2 ppm secondary maximum contaminant level for fluoride in drinking water, its most stringent risk threshold ${ }^{14}$. However, because the signal-to-noise ratio at $0.1 \mathrm{mM} \mathrm{NaF}$ is below 3 , we estimated the reliable lower limit of detection to be $0.2 \mathrm{mM} \mathrm{NaF}$. Importantly, the system also has low leak - we observed minimal activation of gene expression in the absence of NaF. Titration of identical concentrations of $\mathrm{NaCl}$ showed no increase in expression at any condition, demonstrating that the riboswitch is highly specific for fluoride (Figure 1C, grey line). This result corroborates a previous and more extensive characterization in E. coli of the switch's specificity for fluoride ${ }^{12}$. Thus, without any optimization of riboswitch structure or function, the sensor can discriminate health-relevant concentrations of fluoride dosed into laboratory water samples.

\section{Changing reporters to tune sensor speed and detection threshold}

Biosensor field deployment requires an output that can be quickly read with minimal supplemental equipment ${ }^{16}$. Using the maximally activating fluoride concentration $(3.5 \mathrm{mM})$, reactions achieved measurable signal above the no-fluoride $\mathrm{OFF}$ state in 30 minutes at $30^{\circ} \mathrm{C}$, with overall 20-fold activation relative to the no-fluoride condition at the end of the 8-hour experiment (Figure 2A). Despite this, the sensor's ON state was not distinguishable by eye for several hours even after excitation with a blue LED, presenting the need for a faster reporter. 
We hypothesized that we could accelerate the sensor's response with a 3-way junction dimeric Broccoli $(3 \mathrm{WJdB})^{17}$ reporter, an RNA aptamer that activates fluorescence of its DFHBI-1T ligand upon transcription, eliminating delays caused by translation. At all tested $\mathrm{NaF}$ concentrations, $3 \mathrm{WJdB}$ produced a signal detectable over background within 12 minutes at $30^{\circ} \mathrm{C}$ (Figure 2B), more than twice as fast as could be achieved with sfGFP (Figure 2A). Interestingly, this result also confirms that the fluoride riboswitch is compatible with RNA reporters, despite the potential for misfolding with the upstream riboswitch sequence. However, despite the improvement in speed, exchanging sfGFP for $3 \mathrm{WJdB}$ resulted in a 50-fold reduction in the sensor's fluorescent output at the maximally activating tested condition. Thus, although the RNA-level output is preferable for its speed relative to the sfGFP output if a plate reader is accessible, it is not bright enough to use for field deployment.

As an alternative to a fluorescent output, we used the colorimetric enzyme catechol $(2,3)$ dioxygenase $(\mathrm{C} 23 \mathrm{DO})$ as a reporter. $\mathrm{C} 23 \mathrm{DO}$ has previously been used in geneticallyencoded biosensors for plant viruses ${ }^{18}$ and produces a visible reporter output by oxidizing its colorless catechol substrate to the yellow-colored 2-hydroxymuconate semialdehyde ${ }^{19}$. This color change allows gene expression to be read out either by light absorbance at 385 nanometers on a plate reader or by the appearance of a yellow color, visible to the naked eye. All tested fluoride concentrations produced a visible output within 70 minutes at $30^{\circ} \mathrm{C}$, which we empirically defined as an absorbance of 0.8 based on our previous observations (Figure 2C) ${ }^{18}$. Notably, there was only a 20 -minute time separation between the minimally and maximally activating conditions, highlighting the ability of enzymatic reporters to quickly amplify weak signals. Consistent with previous uses of C23DO as a reporter in a cell-free reaction ${ }^{19}$, we observed a decay in the absorbance signal after it reached peak activation, possibly due to 2-hydroxymuconate semialdehyde degradation. This effect does not compromise sensor robustness because differences in activation for an enzymatic reporter are determined by differences in time to observable signal rather than final signal magnitude, which is determined by the amount of substrate supplied. One disadvantage of this strategy is that activation time does not linearly correlate with fluoride concentration, limiting the sensor to only supplying a binary presence/absence result within a specified time window ${ }^{16}$. Despite this, the sensor's sensitivity and low leak make this presence/ absence result diagnostically informative, which combined with the advantages of an easily visualized output and reasonable time to detection made C23DO our reporter of choice for a field-deployable diagnostic.

\section{Reaction tuning and lyophilization towards biosensor field deployment}

We next took steps to optimize our sensor to detect fluoride near the EPA's secondary maximum contaminant limit of $2 \mathrm{ppm}(100 \mu \mathrm{M})$. We obtained a robust ON signal with our original design, but the sensor began to leak without fluoride after 90 minutes (Figure 2C, gray line), complicating detection for trace amounts of fluoride. We attempted to mitigate this problem by reducing the amount of reporter DNA supplied to the reaction from $5 \mathrm{nM}$ to $3 \mathrm{nM}$ to diminish the sensor's output. In doing so, we completely suppressed leak while detecting $100 \mu \mathrm{M} \mathrm{NaF}$ over background (Figure 3A), but at the cost of significantly delaying activation. We could detect as low as $50 \mu \mathrm{M} \mathrm{NaF}$ over background in this leakless sensor, but 
only during an extended incubation that did not reach a visually detectable threshold within six hours.

To solve this dilemma and maintain a practical incubation time, we sought a strategy whereby tests could be interpreted as "ON" only if the yellow color appeared within some externally specified time window. Under these constraints, sensor leak is not a problem as long as the difference in timescale between the ON and OFF state is suitably longer than the test time. To implement this strategy, we increased biosensor DNA concentration to $10 \mathrm{nM}$ and also increased the temperature of the CFE reaction to $37^{\circ} \mathrm{C}$. Under these conditions, activation by $100 \mu \mathrm{M} \mathrm{NaF}$ resulted in a clear color change in sixty minutes with no visible leak in the OFF condition (Figure 3B, Supplemental Figure 2). The same conditions using 3 nM DNA template resulted in no color change within 60 minutes. This result highlights an appreciable advantage afforded by the open reaction environment of cell-free systems: the sensor's limit of detection can be tuned simply by manipulating the reaction time and the DNA concentration of the biosensor.

Recent work demonstrates that CFE reactions can be lyophilized and rehydrated when needed for on-demand biomanufacturing, nucleic acid detection, and educational activities $6,7,20,21$. To expand these applications to point-of-use small molecule detection, we next aimed to demonstrate that fluoride biosensor reactions maintain functionality after being lyophilized. We measured the impact of lyophilization on fluoride detection by lyophilizing reactions containing $10 \mathrm{nM} \mathrm{C23DO}$ reporter plasmid overnight. The reactions were then rehydrated with laboratory grade Milli-Q water (Figure 3C, top) or water containing $1 \mathrm{mM} \mathrm{NaF}$ (Figure 3C, bottom) and incubated at $37^{\circ} \mathrm{C}$. Time-lapse photography shows visible activation within 60 minutes in the $1 \mathrm{mM} \mathrm{NaF}$ condition with no leak observed within 100 minutes in the no-fluoride condition (Supplemental Video 1). This finding, consistent with other recent reports from lyophilized cell-free systems ${ }^{6,7,20,21}$, indicates that sensing by the fluoride riboswitch in CFE reactions is not disrupted by the lyophilization process.

We also tested the viability of lyophilized reactions stored over longer periods of time. After lyophilization, reaction tubes were wrapped in Parafilm and stored in Drierite for 3 months in darkness at room temperature and atmospheric pressure before being removed and rehydrated with laboratory grade Milli-Q water or water containing $1 \mathrm{mM} \mathrm{NaF}$. The sample rehydrated with $1 \mathrm{mM} \mathrm{NaF}$ showed strong activation within one hour, with no leak observed in the no-fluoride condition (Supplemental Figure 3). Interestingly, lyophilization appeared to suppress leak in the no-fluoride condition without impacting the ability to activate expression with fluoride. The maintained viability of reactions after three months indicates that storage in desiccant and light shielding to prevent catechol oxidation are the only requirements for long-term storage of lyophilized cell-free reactions, a crucial step towards field-deployment.

\section{Point-of-use detection of environmental fluoride with a lyophilized biosensor}

Components of environmental water samples, particularly natural ions like sodium, magnesium, or potassium, could poison cell-free reactions upon rehydration. These "matrix effects" would then impede the translation of a sensor from lab experiments to field testing 
and must be accounted for in a field-deployable diagnostic. To test the robustness of our system against matrix effects, we created mock fluoride-containing field samples by sampling water from a municipal tap, Lake Michigan, and an outdoor swimming pool, with Milli-Q water used as a control. NaF was then added to each sample to a final concentration of $1 \mathrm{mM}$. The biosensing reactions were prepared as before and pipetted into PCR tubes (Figure 4A, top) or spotted on BSA-treated chromatography paper (Figure 4A, bottom) before being lyophilized overnight. After lyophilization, reactions were immediately rehydrated with either unaltered mock field sample (-condition) or mock field sample containing $1 \mathrm{mM} \mathrm{NaF}$ (+ condition) and incubated at $37^{\circ} \mathrm{C}$ for one hour. For all fluoridecontaining samples both in tubes and on paper, a color change was observed within one hour, with no color development in any of the no-fluoride conditions. These results confirm that the fluoride biosensor is robust against the unfiltered environmental samples tested and can be used in real-world conditions.

As the culmination of our optimization process, we tested our sensor's ability to accurately classify fluoride-containing samples in the field. We specifically sought to follow a previously published environmental fluoride study that used conventional methods to sample and test publicly available natural and municipal water sources near the Irazu volcano in Cartago, Costa Rica, an area shown to have elevated fluoride levels (Supplemental Figure $4)^{23}$. To do this, we manufactured lyophilized fluoride biosensor reactions and transported them to Costa Rica using our simplified desiccant packaging (Supplemental Figure 5A) for field testing. Sampling regions identified in the previous study ${ }^{23}$, we collected samples in 50 $\mathrm{mL}$ conical tubes and tested for fluoride in batch by adding unprocessed water to lyophilized reactions in PCR tubes via single-use exact volume transfer pipettes (Supplemental Figure $5 B)$.

All field-testing was done onsite in Costa Rica without access to laboratory resources or equipment. Reactions were incubated at approximately $37^{\circ} \mathrm{C}$ by being held in the armpit, with reaction time increased to 5 hours to control for delayed activation caused by the imprecision of body heat incubation and low environmental fluoride concentrations ${ }^{18}$. A strong yellow color developed in every positive control reaction within an hour, confirming robustness to reaction poisoning by potential sample matrix effects (Supplemental Table 2). No activation was observed within 5 hours in any samples with fluoride concentrations less than $50 \mu \mathrm{M}(\sim 1 \mathrm{ppm})$ as measured in cross-validation with a commercial fluoride-sensing electrode. However, a visible color change was observed after 3.5 hours in a water sample collected from a roadside ditch measured to have a fluoride concentration of $60 \mu \mathrm{M}$ (Figure 4B). This delayed activation aligns with our previous characterization in detecting trace concentrations of fluoride below $100 \mu \mathrm{M}$ (Figure 3A). For all samples, the commercial electrode measurement confirmed the conclusions drawn from the cell-free sensors, with no false positives or false negatives observed under any conditions $(n=9)$ (Supplemental Table 2). By accurately detecting levels of fluoride relevant to public health concern thresholds in a real-world water source with minimal supplementary equipment, we have shown that lyophilized fluoride biosensor CFE reactions can be effectively used as low-cost, point-ofuse diagnostics, demonstrating the potential of engineered biosensor elements for small molecule detection in the field. 


\section{Discussion}

In this work, we have demonstrated that a fluoride riboswitch can be implemented in a CFE system to act as a field-deployable diagnostic for environmental water samples. To the best of our knowledge, this is the first demonstration of a cell-free riboswitch-based biosensor that can detect health-relevant small molecules at regulatory levels within the field. Importantly, this work represents a significant improvement in efficacy over commercially available consumer kits (Supplemental Figure 6) and provides significant simplification and cost savings over gold standard electrochemical methods of fluoride detection, which cost hundreds to thousands of dollars and are cumbersome to use even for scientifically skilled operators. In contrast, our biosensors can currently be made for $\$ 0.40 /$ reaction $^{20}$, only require a drop of water, and are robust to temperature variation, enabling incubation with body heat.

A key strength of cell-free biosensing is that biochemical parameters such as cofactor and DNA concentration can be easily tuned to reduce leak and improve dynamic range, which has been a historically difficult challenge for riboswitch engineering in cells. Furthermore, since riboswitches are cis-acting, only one DNA template concentration needs to be tuned per sensor, simplifying the optimization space relative to trans-acting RNA or protein regulators. When optimizing these reactions for the field, we found that reactions lyophilized in PCR tubes had advantages over paper-based reactions, which rapidly dried out even when incubated in sealed, humidified containers. This effect was exacerbated by the longer incubation times required for low analyte concentrations, variabilities in ambient temperature, and the practical difficulty of equipment-free incubation of paper sensors using body heat, making the tube format much more amenable to the challenges of field deployment.

This work also highlights the feasibility of using transcriptional riboswitch-mediated gene expression to convert weak-binding RNA aptamers into functional biosensors. We were surprised to find that the $B$. cereus $c r c B$ riboswitch activated so well in an $E$. coli cell-free lysate system, given the sophisticated nature of its folding mechanism and transcriptional readthrough observed both in vitro and in vivo ${ }^{13,24}$ Transcriptional riboswitches often show weak activation due to the short timescales of their regulatory decision-making, resulting in sensitivities that are kinetically, rather than thermodynamically limited ${ }^{25}$. Coupling transcriptional riboswitches to enzymatic outputs like C23DO can amplify weak signals, since each reporter enzyme turns over multiple molecules of substrate ${ }^{26}$. The combined kinetic mechanism of switching and the signal amplification afforded by a colorimetric reporter resulted in our sensor achieving a limit of detection of $50 \mu \mathrm{M}$, less than half of the lowest previously measured $\mathrm{K}_{\mathrm{D}}$ for any fluoride aptamer ${ }^{27}$. Thus, this work is a powerful example of why considering only thermodynamic binding affinities during aptamer selection can exclude promising, diagnostically relevant sensors.

The strategies we present here could be applied to optimize the performance of a large number of natural riboswitches for the detection of metabolites and ions relevant to environmental and human health monitoring ${ }^{28}$. Additionally, the compatibility of CFE reactions for high-throughput screening ${ }^{29}$ and the simple format of our DNA expression 
construct could be used to characterize the thousands of "orphan" riboswitches that have been bioinformatically identified but bind to unknown ligands ${ }^{30}$. We imagine that these strategies could even be used to re-engineer riboswitches to have novel function ${ }^{31-33}$. As the rules of riboswitch mechanisms are deciphered at deeper levels ${ }^{13,34-36}$, we hope to reach a sufficient understanding to design their functional properties to meet the global needs for field-deployable environmental and health diagnostics.

\section{Materials and Methods}

\section{Plasmid Construction}

Plasmids were assembled using Gibson assembly (New England Biolabs, Cat\#E2611S) and purified using a Qiagen QIAfilter Midiprep Kit (QIAGEN, Cat\#12143). pJBL7025 and pJBL7026 were assembled from pJBL3752. A table of all plasmid sequences can be found in Supplemental Table 1.

\section{Extract Preparation}

Extracts were prepared according to published protocols using sonication and postlysis processing in the Rosetta2 (DE3) pLysS strain ${ }^{10}$. Briefly, cells are plated on a chloramphenicol-selective agar plate and incubated overnight then used to inoculate a $20 \mathrm{~mL}$ overnight starter culture for a $1 \mathrm{~L}$ final culture. This culture is grown to an optical density (OD600) of $3.0 \pm 0.2$ then pelleted and lysed by sonication before centrifugation for 10 minutes at $4^{\circ} \mathrm{C}$ and $12,000 \mathrm{~g}$. After lysis, extracts were incubated with shaking for 80 minutes at $37^{\circ} \mathrm{C}$ and $200 \mathrm{rpm}$ then recentrifuged under the same conditions. The supernatant was injected into a 10K MWCO dialysis cassette (ThermoFisher, 66380) and dialyzed at $4^{\circ} \mathrm{C}$ for three hours before a final centrifugation under the same conditions and snapfreezing in liquid nitrogen.

\section{CFE Experiment}

CFE reactions were prepared according to established protocols ${ }^{10}$. Briefly, reactions are composed of cell extract, a reaction buffer containing NTPs, amino acids, buffering salts, crowding agents, and an energy source, and a mix of template DNA and inducers in an approximately 30/30/40 ratio. Between reactions, the only conditions varied are DNA template and concentration, inducer concentration, and buffering magnesium glutamate concentration, the last of which is optimized by extract. Optimal magnesium glutamate concentration was $20 \mathrm{mM}$ for shelf stability and field deployment experiments and $12 \mathrm{mM}$ for all other data. Little variability was seen in extract performance between batches using the appropriate optimal magnesium concentrations (Supplemental Figure 7).

For an example reaction setup, refer to the Supplemental Experimental Design Spreadsheet. All kinetic CFE reactions were prepared on ice in triplicate at the $10 \mu \mathrm{L}$ scale. $33 \mu \mathrm{L}$ of a mixture containing the desired reaction components was prepared and then $10 \mu \mathrm{L}$ was pipetted into three wells of a 384-well plate (Corning, 3712), taking care to avoid bubbles. Plates were sealed (ThermoScientific, 232701) and kinetic data was monitored on a BioTek Synergy H1m plate reader for sfGFP ( $20 \mathrm{nM}$ reporter plasmid, emission/excitation: 485/520 $\mathrm{nm}$ every five minutes for 8 hours at $30^{\circ} \mathrm{C}$ ), $\mathrm{C} 23 \mathrm{DO}$ (variable reporter plasmid 
concentration, $385 \mathrm{~nm}$ absorbance every 30 seconds for $4-6$ hours at $\left.30^{\circ} \mathrm{C}\right)$, and $3 \mathrm{WJdB}(20$ $\mathrm{nM}$ reporter plasmid, emission/excitation $472 / 507 \mathrm{~nm}$ every 30 seconds for 2 hours at $30^{\circ} \mathrm{C}$ ). C23DO reactions were supplemented with $1 \mathrm{mM}$ catechol and $3 \mathrm{WJdB}$ reactions were supplemented with $20 \mu \mathrm{M}$ DFHBI-1T. For all fluorescence experiments, a no-DNA negative control was prepared in triplicate for every extract being tested. All reported fluorescence values have been baseline-subtracted by the average of three samples from the no-DNA condition. Baseline subtraction was not performed for catechol reactions because reaction progress is determined from time to activation rather than maximal absorbance value. For the data depicted in Figure $1 \mathrm{C}, \mathrm{NaF}$ and $\mathrm{NaCl}$ titrations were performed in separate experiments.

\section{Mean Equivalent Fluorescence Calibration}

Fluorescence measurements were calibrated to a standard curve of fluorescein isothiocyanate (FITC) fluorescence to give standardized fluorescence units of $\mu \mathrm{M}$ equivalent FITC following a previously established procedure ${ }^{37}$. Briefly, serial dilutions were performed from a $50 \mu \mathrm{M}$ stock and prepared in a $\mathrm{pH} 9.5,100 \mathrm{mM}$ sodium borate buffer. Fluorescence values for these samples were read at an excitation wavelength of $485 \mathrm{~nm}$ and emission wavelength of $515 \mathrm{~nm}$ for sfGFP and an excitation wavelength of $472 \mathrm{~nm}$ and emission wavelength of $507 \mathrm{~nm}$ for $3 \mathrm{WJdB}$. These values were then used to calculate a linear conversion factor relating the plate reader's output in arbitrary units to the FITC standard curve.

\section{Lyophilization}

All lyophilization was performed in a Labconco FreeZone 2.5 Liter $-84^{\circ} \mathrm{C}$ Benchtop Freeze Dryer (Cat\# 710201000). A CFE reaction master mix was prepared and split into $20 \mu \mathrm{L}$ aliquots in PCR strip tubes. Tube caps were then pierced with a pin and strips were wrapped in aluminum foil before being flash frozen in liquid nitrogen and lyophilized overnight at 0.04 mbar. After lyophilization, pierced PCR strip tube caps were replaced. Tubes were then sealed with parafilm and placed directly into Drierite (Cat\#11001) for storage at room temperature (Supplemental Figure 5A).

\section{Paper Sensors}

Individual sensors were punched out of Whatman 1 CHR chromatography paper (3001-861) using a Swingline Commercial Desktop Punch (A7074020). Tickets were then placed in a petri dish and immersed in 4\% BSA for one hour before being transferred to a new dish and left to air dry overnight. After drying, tickets were spotted with $20 \mu \mathrm{L}$ of CFE reaction and placed in plastic jars (QOSMEDIX 29258), which were loosely capped and wrapped in aluminum foil before being flash frozen in liquid nitrogen and lyophilized overnight at 0.04 mbar. For testing, tickets were transferred to new jars and rehydrated with $20 \mu \mathrm{L}$ of sample solution. Jars were then closed and sealed with parafilm before incubation for one hour at $37^{\circ} \mathrm{C}$. 


\section{Field Deployment}

$20 \mu \mathrm{L}$ lyophilized reactions were prepared with $10 \mathrm{nM}$ pJBL7025 and $1 \mathrm{mM}$ catechol. As a positive control, additional reactions were lyophilized after being pre-enriched with $1 \mathrm{mM}$ $\mathrm{NaF}$. Supplemental Table 2 contains a complete list of sample site locations and water sources tested. $50 \mathrm{~mL}$ water samples were collected and stored in Falcon tubes (Fisher Scientific, Cat\# 14-432-22) without any processing or filtration. Reactions were rehydrated by using $20 \mu \mathrm{L}$ exact volume transfer pipettes (Thomas Scientific, 1207F80) to pull from collected samples. Three reactions were run at each sample site: (1) a positive control rehydrated with the sample, (2) a blank reaction rehydrated with the sample, and (3) a negative control reaction rehydrated with purified water to test for any reaction leak. Reactions were placed in a plastic bag and incubated at body temperature in the armpit for five hours using established protocols and marked as activated if a visible yellow color was observed $^{18}$. Quantitative measurements of fluoride concentration of the same sample were taken with an Extech ExStik Waterproof Fluoride Meter (Cat\# FL700).

\section{Image Capture}

All images were captured with via cell phone camera, with no specialized photography setup and no post-capture editing done aside from cropping image borders. Tubes were illuminated from below via desk lamp to highlight reaction color change. Paper sensors were illuminated from above via desk lamp and photographed without removal from the plastic jars used for incubation.

\section{Supplementary Material}

Refer to Web version on PubMed Central for supplementary material.

\section{Acknowledgements}

We would like to thank Professor Ana Gabriela Calderón Cornejo (Universidad de Costa Rica) and Eduardo Quirós Morales for assistance with biosensor field-testing. We also thank Ashty Karim (Northwestern University) and Professor Robert Batey (University of Colorado, Boulder) for helpful comments in preparing the manuscript, along with Khalid Alam (Stemloop, Inc.) for editing the supplemental video, Jaeyoung Jung (Northwestern University) for assistance with designing the graphical abstract, and Thomas Shahady (University of Lynchburg) for helpful comments about water sampling in Costa Rica. This work was supported by the Air Force Research Laboratory Center of Excellence for Advanced Bioprogrammable Nanomaterials (C-ABN) Grant FA8650-15-2-5518 (to M.C.J. and J.B.L), the David and Lucile Packard Foundation (to M.C.J.), an NSF CAREER Award (1452441 to J.B.L.), and the Camille Dreyfus Teacher-Scholar Program (to M.C.J. and J.B.L.). A.D.S. was supported in part by the National Institutes of Health Training Grant (T32GM008449) through Northwestern University's

Biotechnology Training Program. The views and conclusions contained herein are those of the authors and should not be interpreted as necessarily representing the official policies or endorsements, either expressed or implied, of the Air Force Research Laboratory, Air Force Office of Scientific Research, or US Government.

\section{References}

(1). World Health Organization. World Health Statistics 2016: Monitoring Health for the SDGs Sustainable Development Goals; World Health Organization, 2016.

(2). Onda K; LoBuglio J; Bartram J. Global Access to Safe Water: Accounting for Water Quality and the Resulting Impact on MDG Progress. Int. J. Environ. Res. Public Health 2012, 9 (3), 880-894. [PubMed: 22690170]

(3). Maheshwari RC Fluoride in Drinking Water and Its Removal. J. Hazard. Mater 2006, 137 (1), 456-463. [PubMed: 16600479] 
(4). World Health Organization. Guidelines for Drinking-Water Quality. WHO Chron. 2011, 38 (4), 104-108.

(5). Zhou Y; Zhang JF; Yoon J. Fluorescence and Colorimetric Chemosensors for Fluoride-Ion Detection. Chem. Rev 2014, 114 (10), 5511-5571. [PubMed: 24661114]

(6). Pardee K; Green AA; Ferrante T; Cameron DE; DaleyKeyser A; Yin P; Collins JJ Paper-Based Synthetic Gene Networks. Cell 2014, 159 (4), 940-954. [PubMed: 25417167]

(7). Pardee K; Green AA; Takahashi MK; Braff D; Lambert G; Lee JW; Ferrante T; Ma D; Donghia N; Fan M. Rapid, Low-Cost Detection of Zika Virus Using Programmable Biomolecular Components. Cell 2016, 165 (5), 1255-1266. [PubMed: 27160350]

(8). Gräwe A; Dreyer A; Vornholt T; Barteczko U; Buchholz L; Drews G; Ho UL; Jackowski ME; Kracht M; Lüders J. A Paper-Based, Cell-Free Biosensor System for the Detection of Heavy Metals and Date Rape Drugs. PLoS One 2019, 14 (3), e0210940.

(9). Gupta S; Sarkar S; Katranidis A; Bhattacharya J. Development of a Cell-Free Optical Biosensor for Detection of a Broad Range of Mercury Contaminants in Water: A Plasmid DNA-Based Approach. ACS Omega 2019, 4 (5), 9480-9487. [PubMed: 31460039]

(10). Silverman A; Kelley-Loughnane N; Lucks JB; Jewett MC Deconstructing Cell-Free Extract Preparation for in Vitro Activation of Transcriptional Genetic Circuitry. ACS Synth. Biol 2018, 8 (2), 403-414.

(11). Carlson ED; Gan R; Hodgman CE; Jewett MC Cell-Free Protein Synthesis: Applications Come of Age. Biotechnol. Adv 2012, 30 (5), 1185-1194. [PubMed: 22008973]

(12). Baker JL; Sudarsan N; Weinberg Z; Roth A; Stockbridge RB; Breaker RR Widespread Genetic Switches and Toxicity Resistance Proteins for Fluoride. Science (80. ). 2012, 335 (6065), 233235.

(13). Watters KE; Strobel EJ; Angela MY; Lis JT; Lucks JB Cotranscriptional Folding of a Riboswitch at Nucleotide Resolution. Nat. Struct. Mol. Biol 2016, 23 (12), 1124. [PubMed: 27798597]

(14). Doull J; Boekelheide K; Farishian BG; Isaacson RL; Klotz JB; Kumar JV; Limeback H; Poole C; Puzas JE; Reed NMR Fluoride in Drinking Water: A Scientific Review of EPA's Standards. Natl. Acad. Washingt 2006, 205-223.

(15). Zadeh JN; Steenberg CD; Bois JS; Wolfe BR; Pierce MB; Khan AR; Dirks RM; Pierce NA NUPACK: Analysis and Design of Nucleic Acid Systems. J. Comput. Chem 2011, 32 (1), 170 173. [PubMed: 20645303]

(16). McNerney MP; Zhang Y; Steppe P; Silverman AD; Jewett MC; Styczynski MP Point-of-Care Biomarker Quantification Enabled by Sample-Specific Calibration. Sci. Adv 2019, 5 (9), eaax4473.

(17). Alam KK; Tawiah KD; Lichte MF; Porciani D; Burke DH A Fluorescent Split Aptamer for Visualizing RNA-RNA Assembly In Vivo. ACS Synth. Biol 2017, 6 (9), 1710-1721. [PubMed: 28548488]

(18). Verosloff M; Chappell J; Perry KL; Thompson JR; Lucks JB PLANT-Dx: A Molecular Diagnostic for Point-of-Use Detection of Plant Pathogens. ACS Synth. Biol 2019, 8 (4), 902 905. [PubMed: 30790518]

(19). Chappell J; Westbrook A; Verosloff M; Lucks JB Computational Design of Small Transcription Activating RNAs for Versatile and Dynamic Gene Regulation. Nat. Commun 2017, 8 (1), 1051. [PubMed: 29051490]

(20). Stark JC; Huang A; Nguyen PQ; Dubner RS; Hsu KJ; Ferrante TC; Anderson M; Kanapskyte A; Mucha Q; Packett JS; et al. BioBitsTM Bright: A Fluorescent Synthetic Biology Education Kit. Sci. Adv 2018, 4 (8), eaat5107.

(21). Huang A; Nguyen PQ; Stark JC; Takahashi MK; Donghia N; Ferrante T; Dy AJ; Hsu KJ; Dubner RS; Pardee K; et al. BioBitsTM Explorer: A Modular Synthetic Biology Education Kit. Sci. Adv 2018, 4 (8), eaat5105.

(22). Haklay M; Weber P. Openstreetmap: User-Generated Street Maps. Ieee Pervas Comput 2008, 7 (4), 12-18.

(23). Rojas Zuniga F; Floor G; Malavassi E; Martinez Cruz M; Van Bergen M. Fluorosis Dental En La Población Infantil En Las Cercanías Del Volcán Irazú, Costa Rica. Congr. Latinoam. Estud. Química Paraguay 2014. 
(24). Zhao B; Guffy SL; Williams B; Zhang Q. An Excited State Underlies Gene Regulation of a Transcriptional Riboswitch. Nat. Chem. Biol 2017, 13 (9), 968-974. [PubMed: 28719589]

(25). Wickiser JK; Winkler WC; Breaker RR; Crothers DM. The Speed of RNA Transcription and Metabolite Binding Kinetics Operate an FMN Riboswitch. Mol. Cell 2005, 18 (1), 49-60. [PubMed: 15808508]

(26). Karzbrun E; Shin J; Bar-Ziv RH; Noireaux V. Coarse-Grained Dynamics of Protein Synthesis in a Cell-Free System. Phys. Rev. Lett 2011, 106 (4), 48104.

(27). Ren A; Rajashankar KR; Patel DJ Fluoride Ion Encapsulation by Mg 2+ Ions and Phosphates in a Fluoride Riboswitch. Nature 2012, 486 (7401), 85. [PubMed: 22678284]

(28). McCown PJ; Corbino KA; Stav S; Sherlock ME; Breaker RR Riboswitch Diversity and Distribution. RNA 2017, 23 (7), 995-1011. [PubMed: 28396576]

(29). Moore SJ; MacDonald JT; Wienecke S; Ishwarbhai A; Tsipa A; Aw R; Kylilis N; Bell DJ; McClymont DW; Jensen K; et al. Rapid Acquisition and Model-Based Analysis of Cell-Free Transcription-Translation Reactions from Nonmodel Bacteria. Proc. Natl. Acad. Sci 2018, 115 (19).

(30). Greenlee EB; Stav S; Atilho RM; Brewer KI; Harris KA; Malkowski SN; Mirihana Arachchilage G; Perkins KR; Sherlock ME; Breaker RR Challenges of Ligand Identification for the Second Wave of Orphan Riboswitch Candidates. RNA Biol 2018, 15 (3), 377-390. [PubMed: 29135333]

(31). Boussebayle A; Torka D; Ollivaud S; Braun J; Bofill-Bosch C; Dombrowski M; Groher F; Hamacher K; Suess B. Next-Level Riboswitch Development- Implementation of CaptureSELEX Facilitates Identification of a New Synthetic Riboswitch. Nucleic Acids Res. 2019, 47 (9), 4883-4895. [PubMed: 30957848]

(32). Espah Borujeni A; Mishler DM; Wang J; Huso W; Salis HM Automated Physics-Based Design of Synthetic Riboswitches from Diverse RNA Aptamers. Nucleic Acids Res. 2015, 44 (1), 1-13. [PubMed: 26621913]

(33). Wu MJ; Andreasson JOL; Kladwang W; Greenleaf WJ; Das R. Automated Design of Diverse Stand-Alone Riboswitches. ACS Synth. Biol 2019, 8 (8), 1838-1846. [PubMed: 31298841]

(34). Frieda KL; Block SM Direct Observation of Cotranscriptional Folding in an Adenine Riboswitch. Science (80-. ). 2012, 338 (6105), 397-400.

(35). Drogalis LK; Batey RT Requirements for Efficient Cotranscriptional Regulatory Switching in Designed Variants of the Bacillus Subtilis PbuE Adenine-Responsive Riboswitch. bioRxiv 2018 $10.1101 / 372573$

(36). Strobel EJ; Cheng L; Berman KE; Carlson PD; Lucks JB A Ligand-Gated Strand Displacement Mechanism for ZTP Riboswitch Transcription Control. Nat. Chem. Biol 2019, 15 (11), 10671076. [PubMed: 31636437]

(37). Alam KK; Jung JK; Verosloff MS; Clauer PR; Lee JW; Capdevila DA; Pastén PA; Giedroc DP; Collins JJ; Lucks JB Rapid, Low-Cost Detection of Water Contaminants Using Regulated In Vitro Transcription. bioRxiv 2019 10.1101/619296 
a
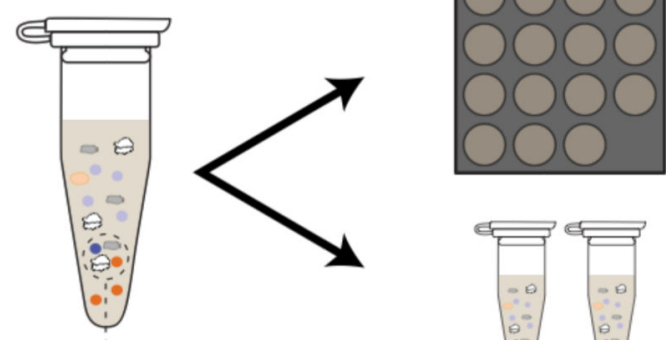

b
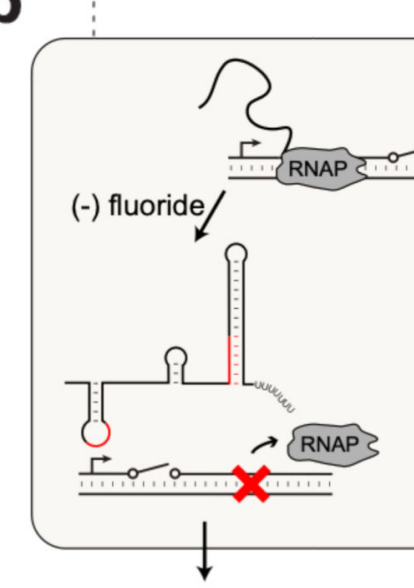

OFF

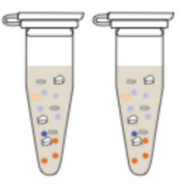

lyophilize

rehydrate
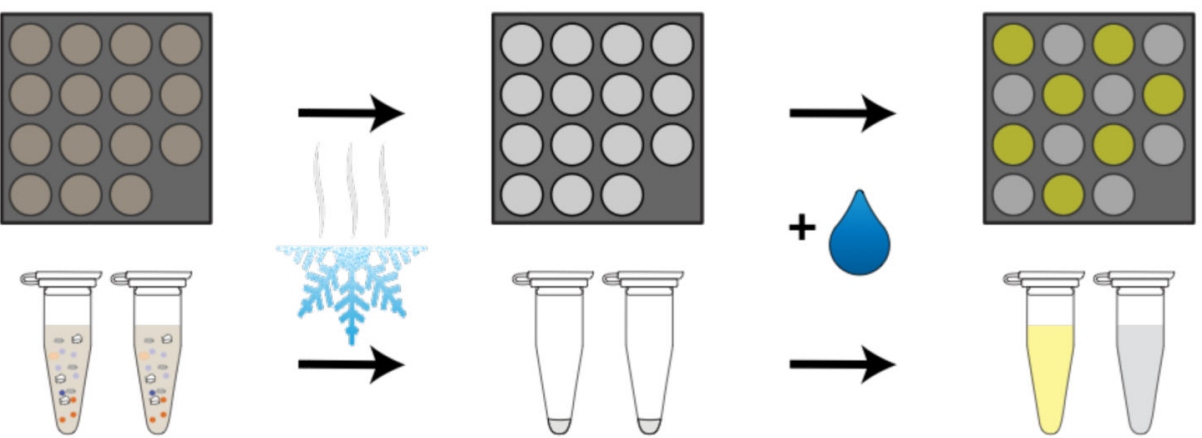

C

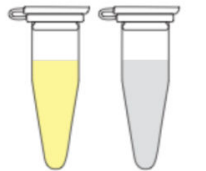

$P_{\text {J23119 }} F^{-} \quad$ RBS

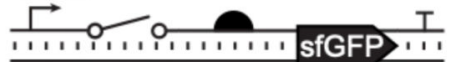

(20 $\mathrm{nM}$ reporter plasmid)
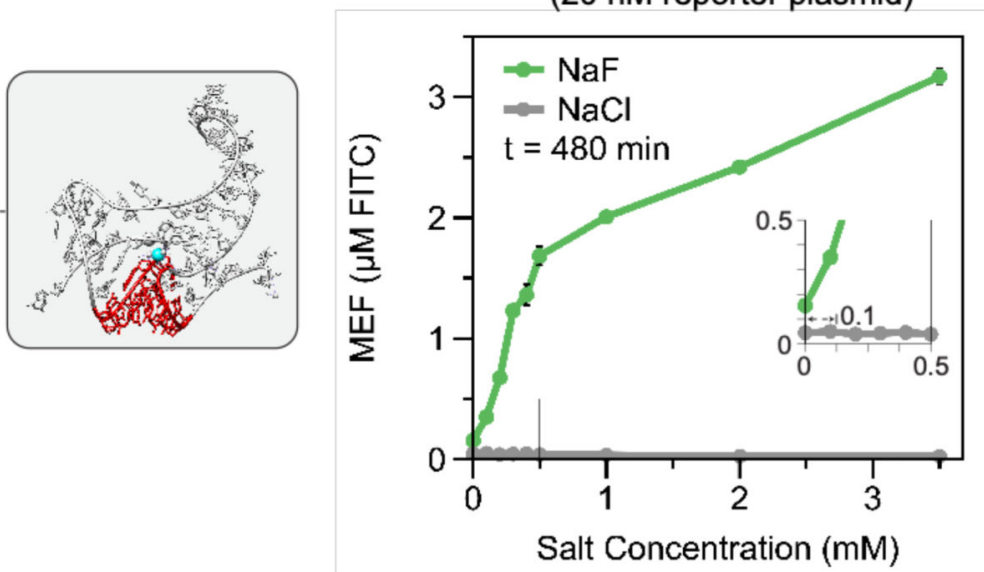

Figure 1. Cell-free fluoride biosensor engineering strategy.

(a) Schematic for lyophilization of a cell-free reaction in tubes or on paper disks.

Rehydration with a water sample allows the designed biosensing reaction to proceed to yield a detectable signal. (b) Schematic for fluoride riboswitch-mediated transcriptional regulation in cell-free extract. The riboswitch folds cotranscriptionally into one of two mutually exclusive states, depending on the presence of fluoride. In the absence of fluoride, the riboswitch folds into a terminating hairpin, precluding downstream gene expression. Fluoride binding stabilizes a pseudoknot structure (red paired region, inset from PDB: $4 \mathrm{ENC}$ ) that sequesters the terminator and enables the expression of downstream reporter genes. (c) Schematic of a cell-free fluoride biosensor, consisting of a DNA template encoding the fluoride riboswitch controlling the expression of sfGFP. Eight-hour endpoint fluorescence measurements for reactions containing $\mathrm{NaF}$ (dark green) or $\mathrm{NaCl}$ (gray) are shown below. Error bars represent one standard deviation from three technical replicates. 
a
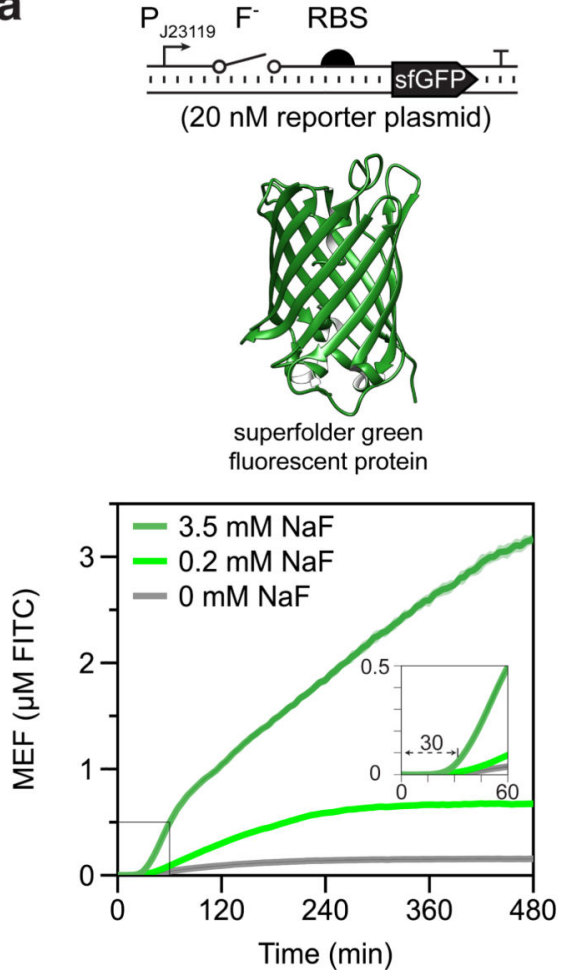

b
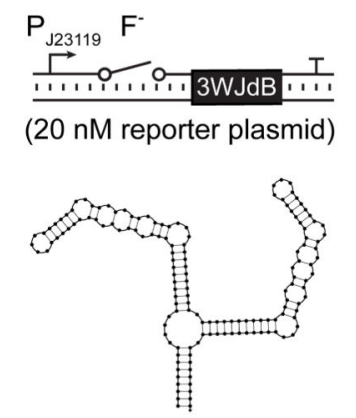

3-way junction dimeric broccoli

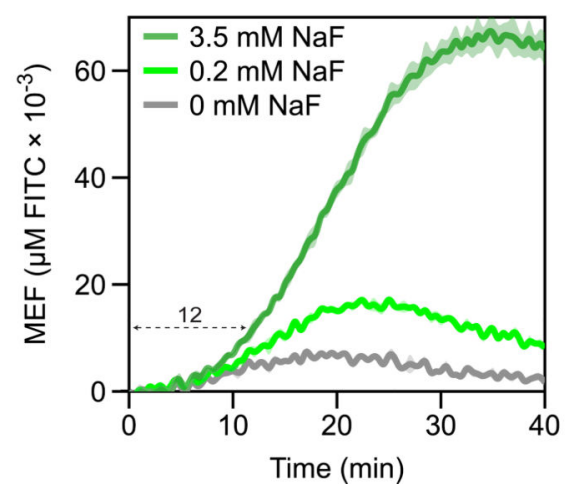

C
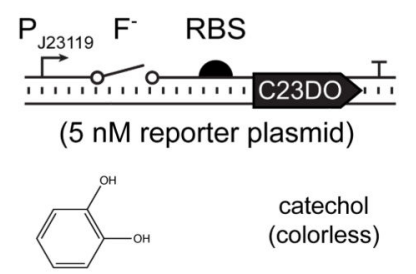

catechol
(colorless)
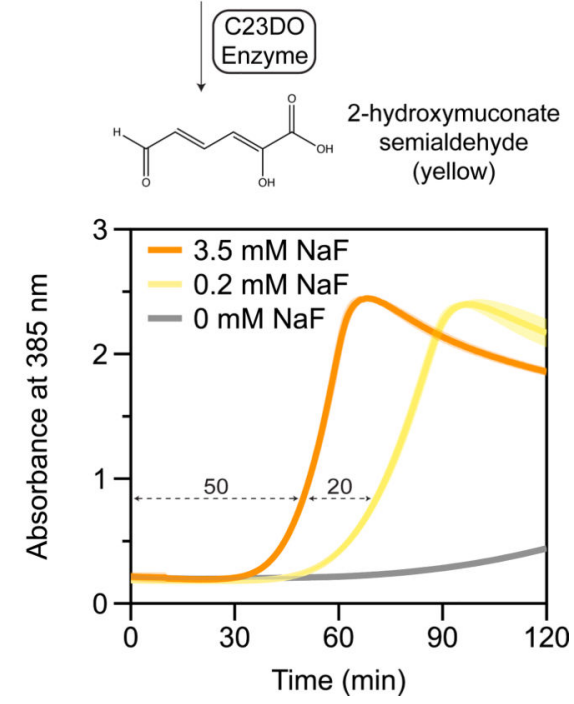

Figure 2. Riboswitch modularity allows fluorescent protein, RNA aptamer and enzymatic colorimetric reporter outputs.

Biosensor DNA template layouts and concentrations shown above reporter information and characterization data for that reporter. (a) Superfolder GFP (sfGFP) reporter (structure from PDB: 2B3P). Time course of fluorescence in the presence of $3.5 \mathrm{mM} \mathrm{NaF}$ (dark green), 0.2 $\mathrm{mM} \mathrm{NaF}$ (light green), or $0 \mathrm{mM} \mathrm{NaF}$ (gray). (b) 3-way junction dimeric Broccoli reporter (structure predicted from NUPACK ${ }^{15}$ ). Time course of fluorescence in the presence of 3.5 $\mathrm{mM} \mathrm{NaF}$ (dark green), $0.2 \mathrm{mM} \mathrm{NaF}$ (light green) and $0 \mathrm{mM} \mathrm{NaF}$ (gray). (c) Catechol $(2,3)$ dioxygenase $(\mathrm{C} 23 \mathrm{DO})$ reporter. Reaction scheme shows the cleavage of the colorless catechol molecule into the yellow 2-hydroxymuconate semialdehyde. Time course of absorbance at $385 \mathrm{~nm}$ in the presence of $3.5 \mathrm{mM} \mathrm{NaF}$ (orange), $0.2 \mathrm{mM} \mathrm{NaF}$ (yellow), and 0 $\mathrm{mM} \mathrm{NaF}$ (gray). For each plot, trajectories represent average and error shading represents one standard deviation from three technical replicates. (a) and (b) are reported in mean equivalent fluorescence (MEF). 

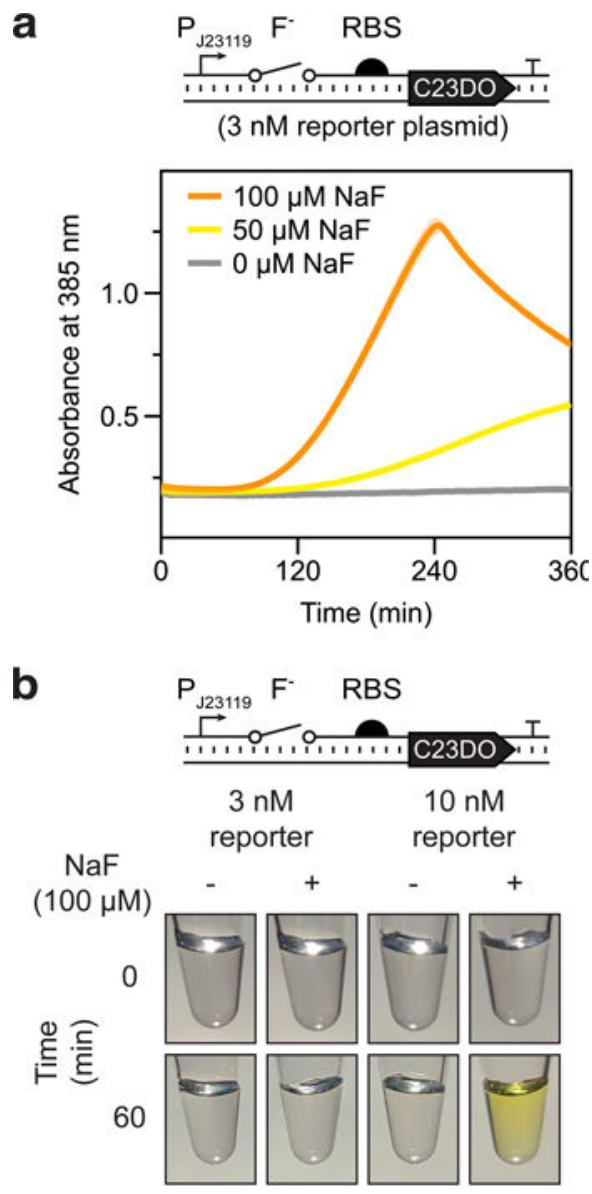

C

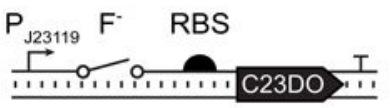

(10 $\mathrm{nM}$ reporter plasmid) Lyophilized - 1 day storage

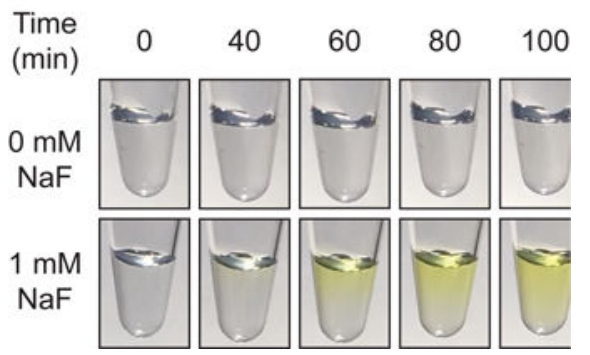

Figure 3. Colorimetric reporters enable fluoride sensing at environmentally relevant concentrations.

(a) Time course of $385 \mathrm{~nm}$ absorbance as measured by plate reader in the presence of 100 $\mu \mathrm{M} \mathrm{NaF}$ (orange), $50 \mu \mathrm{M} \mathrm{NaF}$ (yellow), and $0 \mu \mathrm{M} \mathrm{NaF}$ (grey) using C23DO as a reporter and incubated at $30^{\circ} \mathrm{C}$. Trajectories represent average and error shading represents one standard deviation from three technical replicates. (b) Color change observed after 1-hour for two different reporter template concentrations with and without $100 \mu \mathrm{M} \mathrm{NaF}$. Tubes were mixed by pipetting and incubated at $37^{\circ} \mathrm{C}$ before image capture at 60 minutes. (c) Time 
lapse of rehydrated lyophilized reactions incubated at $37^{\circ} \mathrm{C}$ in the absence (top) and presence (bottom) of $1 \mathrm{mM} \mathrm{NaF}$. 
a

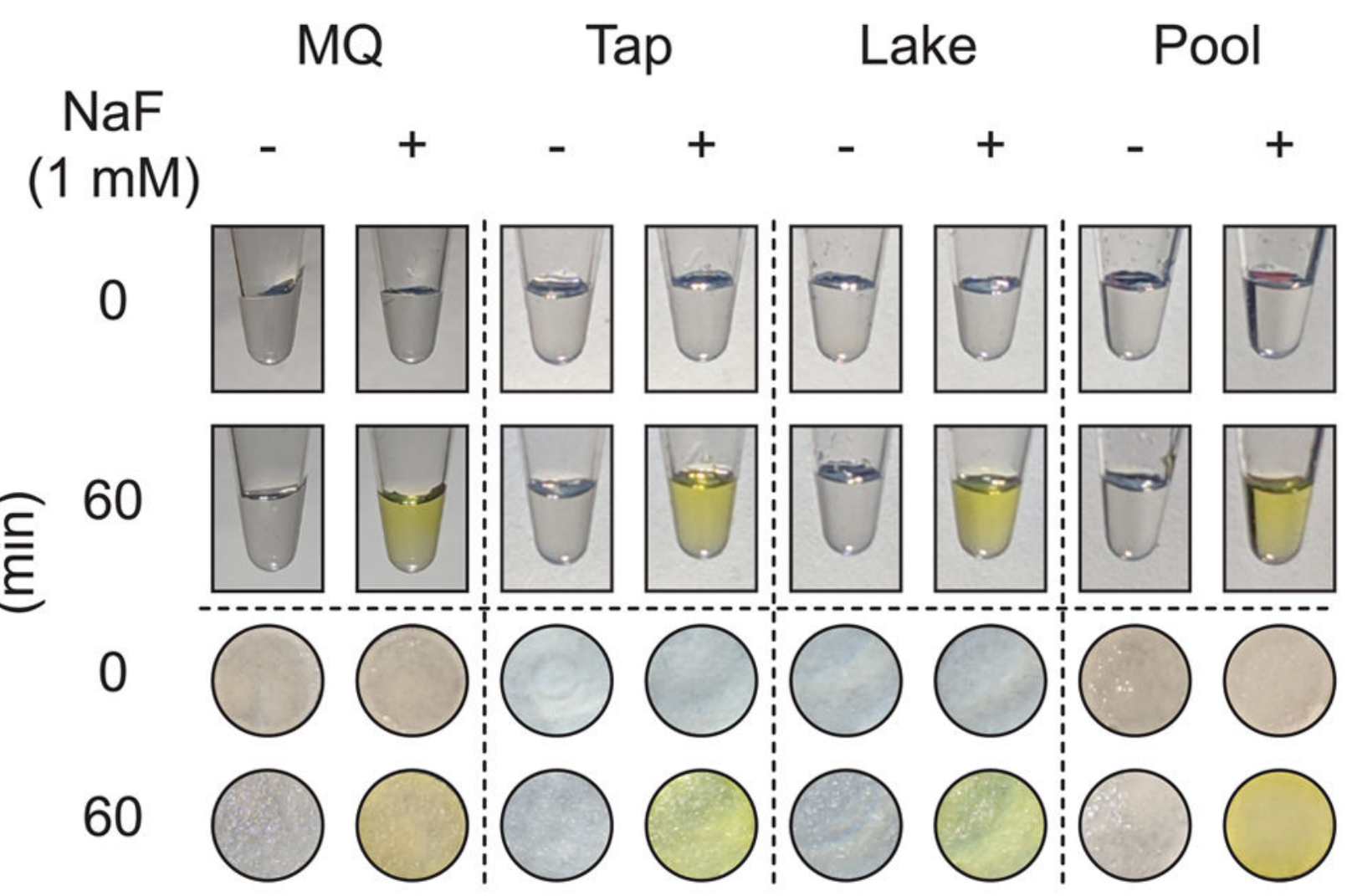

b

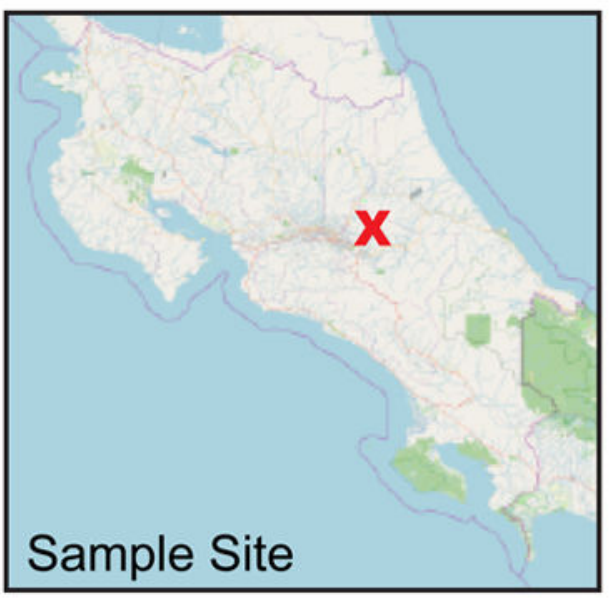

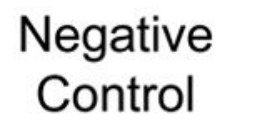

Positive Control

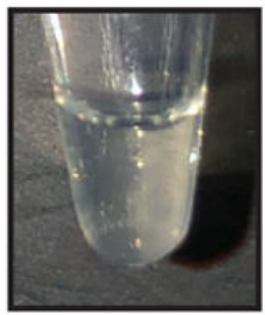

0

Supplied NaF $(\mu \mathrm{M})$

1000

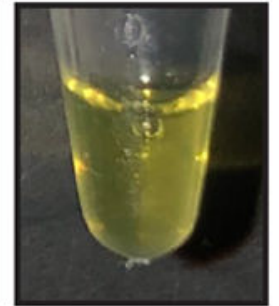

15

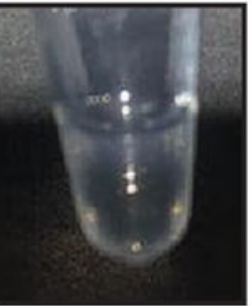

Field

Samples

Measured $\left[\mathrm{F}^{-}\right](\mu \mathrm{M})$

Figure 4. The cell-free fluoride riboswitch biosensor functions with real-world water samples and is not impacted by long-term storage and distribution.

(a) Cell-free reactions rehydrated with various water samples with or without $1 \mathrm{mM} \mathrm{NaF}$ added. Lyophilized reactions in tubes are shown above lyophilized reactions on chromatography paper before and after one-hour incubation at $37^{\circ} \mathrm{C}$. MQ = laboratory grade Milli-Q water; Tap = tap water; Lake $=$ unfiltered Lake Michigan water; Pool $=$ unfiltered outdoor pool water. Uncropped photos of chromatography paper experiments are available in Supplemental Figure 8. (b) Field testing of lyophilized cell-free reactions rehydrated with 
water sampled in Cartago, Costa Rica. Geographical map from OpenStreetMap ${ }^{22}$. The positive control contained $1 \mathrm{mM} \mathrm{NaF}$ in the reaction before lyophilization. The negative control was rehydrated with Milli-Q water, and the positive control and each test were rehydrated with $20 \mu \mathrm{L}$ of unprocessed field sample followed by body-heat incubation for five hours. Measured fluoride concentrations obtained using a fluoride sensing electrode. Field samples are from sites B and E in Supplementary Table 2. 